\title{
openheart Risk stratification of adults with congenital heart disease during the COVID-19 pandemic: insights from a multinational survey among European experts
}

Francisco Javier Ruperti-Repilado (10 , ${ }^{1}$ Daniel Tobler, ${ }^{2}$ Matthias Greutmann, ${ }^{3}$ Judith Bouchardy, ${ }^{4,5}$ Magalie Ladouceur, ${ }^{6}$ Laura Dos-Subira (D) ,' Pastora Gallego (D) , ${ }^{8}$ Harald Gabriel, ${ }^{9}$ Berto Bouma, ${ }^{10}$ Markus Schwerzmann (D) , on behalf of EPOCH

- Prepublication history and additional material are published online only. To view please visit the journal online (http://dx.doi. org/10.1136/openhrt-2020001455).

To cite: Ruperti-Repilado FJ, Tobler D, Greutmann M, et al. Risk stratification of adults with congenital heart disease during the COVID-19 pandemic: insights from a multinational survey among European experts. Open Heart 2021;8:e001455. doi:10.1136/ openhrt-2020-001455

Received 18 September 2020 Revised 9 March 2021 Accepted 22 March 2021
Check for updates

(C) Author(s) (or their employer(s)) 2021. Re-use permitted under CC BY-NC. No commercial re-use. See rights and permissions. Published by BMJ.

For numbered affiliations see end of article.

Correspondence to Dr Markus Schwerzmann; markus.schwerzmann@insel.ch

\section{ABSTRACT}

Objective Adults with congenital heart disease (ACHD) may be at a higher risk of a fatal outcome in case of COVID-19. Current risk stratification among these patients relies on personal experience and extrapolation from patients with acquired heart disease. We aimed to provide an expert view on risk stratification while awaiting results from observational studies.

Methods This study was an initiative of the EPOCH (European Collaboration for Prospective Outcome Research in Congenital Heart disease). Among nine European countries (Austria, Belgium, Denmark, France, Germany, Italy, the Netherlands, Spain and Switzerland), 24 experts from 23 tertiary ACHD centres participated in the survey. ACHD experts were asked to identify ACHD-specific COVID-19 risk factors from a list of potential outcome predictors and to estimate the risk of adverse COVID-19 outcomes in seven commonly seen patient scenarios. Results $82 \%$ of participants did not consider all ACHD patients at risk of COVID-19 related complications. There was a consensus on pulmonary arterial hypertension, Fontan physiology and cyanotic heart disease as risk factors for adverse outcomes. Among different ACHD scenarios, a patient with Eisenmenger syndrome was considered to be at the highest risk. There was a marked variability in risk estimation among the other potential outcome predictors and ACHD scenarios.

Conclusions Pulmonary arterial hypertension, Fontan palliation and cyanotic heart disease were widely considered as risk factors for poor outcome in COVID-19. However, there was a marked disparity in risk estimation for other clinical scenarios. We are in urgent need of outcome studies in ACHD suffering from COVID-19.

\section{INTRODUCTION}

COVID-19 has developed into a worldwide pandemic. ${ }^{1}$ As a response, strategies were developed to minimise transmission of the virus and reduce disease-related morbidity

\section{Key questions}

What is already known about this subject?

- The novel SARS-CoV-2 responsible for COVID-19 is thought to interact with the cardiovascular system on multiple levels, leading to increased morbidity and mortality in patients with underlying cardiovascular diseases.

What does this study add?

- Whether adults with congenital heart disease (ACHD) should be considered to be at increased risk of poor outcomes if suffering from COVID-19 is unclear.

- In the absence of observational studies, our survey provides the first expert opinion in this respect.

How might this impact on clinical practice?

- Our study provides a first impression on how risk stratification of ACHD in the current pandemic is handled in the absence of observational data.

- The results presented in this manuscript may encourage ACHD professionals worldwide to collaborate in outcome studies reflecting the disease among these patients.

and mortality, including preferential protection of patients with underlying medical conditions.

Adults congenital heart disease (ACHD) patients may be such vulnerable persons. Due to their young age (the median age in European registries varies between 30 and 35 years), ${ }^{2}{ }^{3}$ cardiovascular risk factors for adverse COVID-19 outcomes such as ischaemic heart disease, cerebrovascular disease, diabetes and hypertension are infrequently found among ACHD patients. However, many ACHD patients face a lifelong increased risk of arrhythmias, heart failure, paradoxical embolism and pulmonary vascular disease 
due to residual haemodynamic lesions or sequelae from previous interventions. ${ }^{45}$ Therefore, risk stratification among this rapidly growing cohort of rather young and otherwise healthy patients can be challenging.

Currently, risk estimation in ACHD is based on expert opinion. ${ }^{6-8}$ To further delineate COVID-19 risk perception among ACHD specialists, we conducted a survey among 23 tertiary European centres in different countries. The survey allows to identify areas of concordance and discordance regarding COVID-19 risk stratification in ACHD patients.

\section{METHODS}

This survey was an initiative of the EPOCH (European Collaboration for Prospective Outcome Research in Congenital Heart disease; https://www.sacher-registry. com/epoch/). Board members of the European Society of Cardiology ACHD Working Group were invited to participate and identify additional tertiary ACHD centres in their countries. Twenty-three centres from Austria, Belgium, Denmark, France, Germany, Italy, the Netherlands, Spain and Switzerland participated in an online questionnaire using the platform SurveyMonkey. The questionnaire is provided as online supplemental material. All participating ACHD centres were tertiary care hospitals with intensive care units and the possibility to provide extracorporeal membrane oxygenation if necessary.

Participants were asked whether they considered all ACHD patients to be at risk of COVID-19-related complications or not and whether their standards for risk stratification were based on national or centre-specific agreement or on personal judgement. Participants then had to select general and ACHD-specific risk factors they considered as relevant for poor COVID-19-related outcome. Finally, participants estimated the risk of adverse COVID-19 outcomes in seven different common patient scenarios by scoring risks from 0 (no increased risk) to 100 (very high risk). Scores $<25$ were categorised as low risk, scores from 25 to 49 as low to moderate risk, scores from 50 to 74 as moderate to high risk, and scores $\geq 75$ as high risk. Data from 24 questionnaires were collected from 5 to 20 April 2020. The results of the survey were analysed by descriptive statistics and are presented as mean $\pm \mathrm{SD}$, median and IQR, or percentage, as appropriate. For the different ACHD scenarios, categorised risk scores (as described above) among ACHD specialists with clinical experience with ACHD COVID-19 cases and those without were compared by means of $\chi^{2}$ test. Overall, the null hypothesis was rejected for $\mathrm{p}$ values $<0.05$.

\section{Patient and public involvement}

There was no public or patient involvement in this survey.

\section{RESULTS}

The mean number of patients under follow-up per year among the reporting centres was $1673 \pm 877$. A median of 2 (1-4) full-time ACHD cardiologists were working per centre. At the time of the survey, 13 $(57 \%)$ centres already had clinical experience with COVID-19 among their ACHD patients.

The majority $(82 \%)$ of participants considered not all ACHD patients at risk of COVID-19-related complications. Overall, $75 \%$ of the participants based their decision on national/working group consensus, while $58 \%$ and $29 \%$ based their decision on personal judgement and centre-specific agreement, respectively. Among the general risk factors for adverse outcomes in COVID-19, all participants considered advanced age, symptomatic heart failure and immunosuppression as such (figure 1). Advanced lung and renal disease, as well as diabetes, were also considered as relevant for ACHD patients by the majority of the experts. There was less agreement with respect to male gender, coronary artery disease, arterial hypertension and advanced liver disease. Pulmonary arterial hypertension was unanimously considered

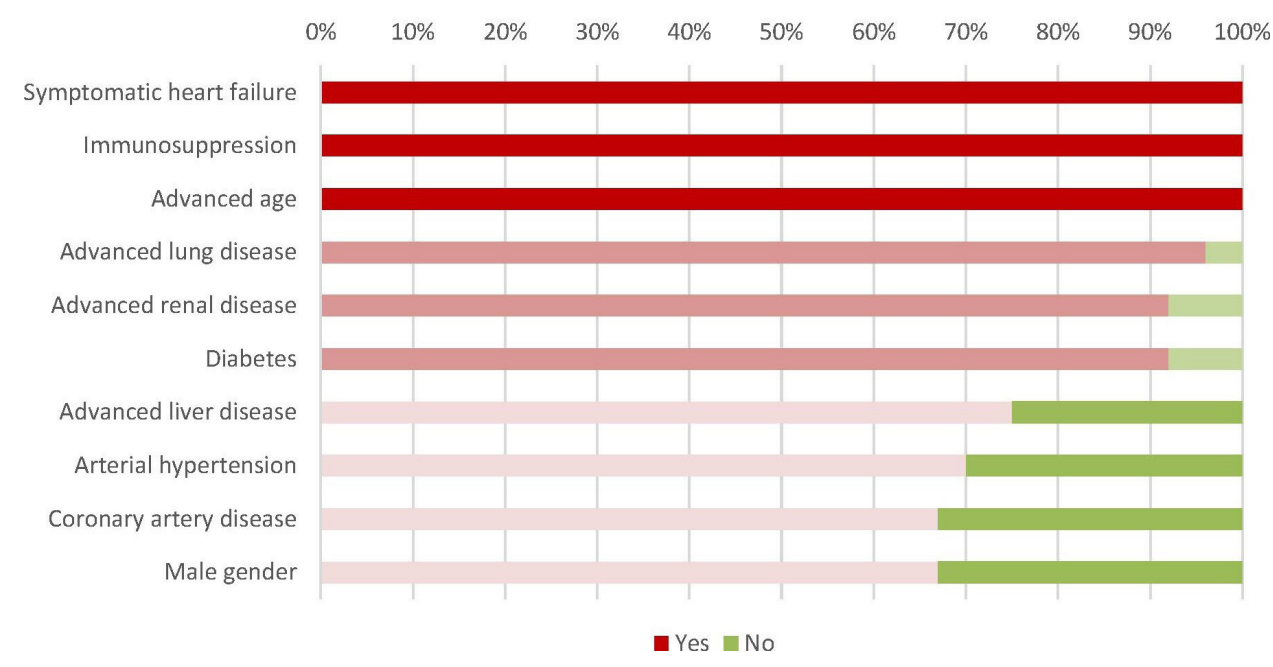

Figure 1 General risk factors (proportion of 'yes' votes) 


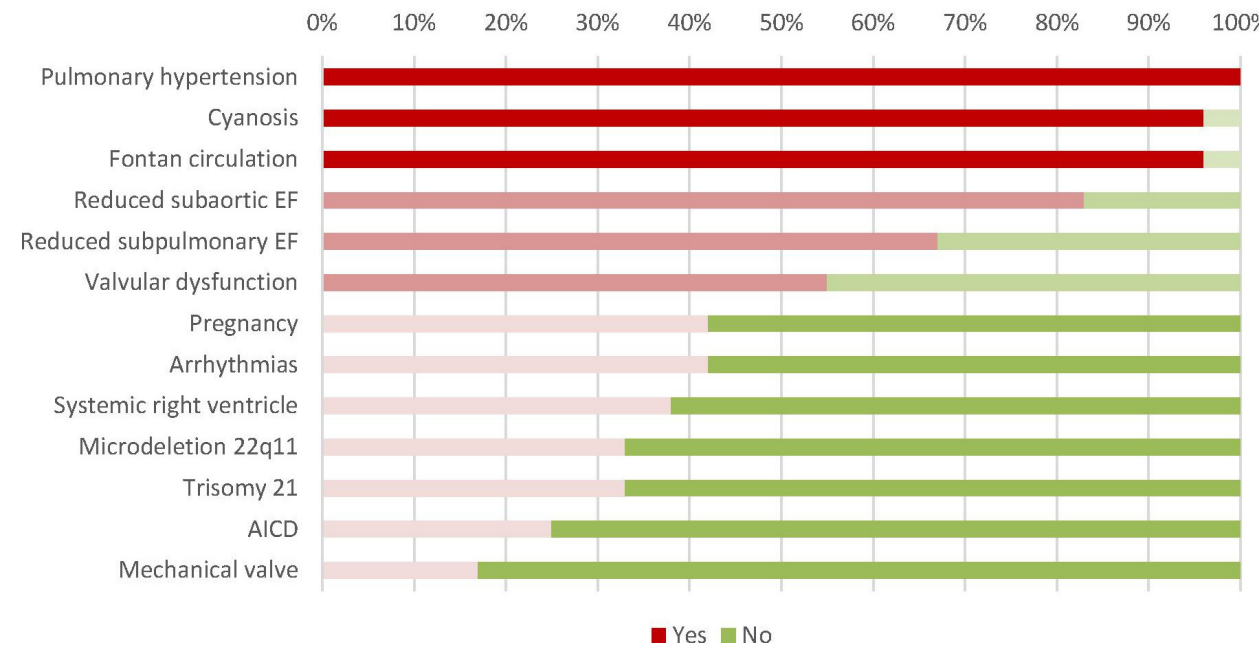

Figure 2 ACHD-specific risk factors (proportion of 'yes' votes). ACHD, adult with congenital heart disease; AICD, automatic implantable cardioverter defibrillator; EF, ejection fraction.

as ACHD-specific risk factor, and 96\% considered Fontan physiology and cyanotic heart disease as additional risk factors. The rating of all proposed general and ACHD-specific risk factors is depicted in figures 1 and 2. Among the different ACHD scenarios, a patient with Eisenmenger syndrome was considered to be at the highest risk, whereas a well-doing Fontan patient with an extracardiac conduit was scored at moderate to high risk. There was a marked variability in risk estimation for this scenario. One-third considered a well-doing Fontan patient to be at high risk for lesion (score $>75$ ), one-third at moderate to high risk (score 50-75) and one-third at low to moderate risk (score 25-49). All other scenarios were scored at low to moderate risk. The SD of the score among all scenarios ranged from 14 to 21 (figure 3).
Complexity of the heart defect and comorbidities were considered the most important risk factor for an adverse outcome in case of COVID-19 (for 33\% and $29 \%$ of the responders, respectively).

For the different ACHD scenarios, comparison of categorised risk scores among ACHD specialists with clinical experience with ACHD COVID-19 cases and those without revealed no statistically significant difference in risk stratification.

\section{DISCUSSION}

In the absence of observational studies, our survey provides the first systematically collected expert opinion on risk stratification of ACHD patients. Those with pulmonary arterial hypertension, Fontan palliation and

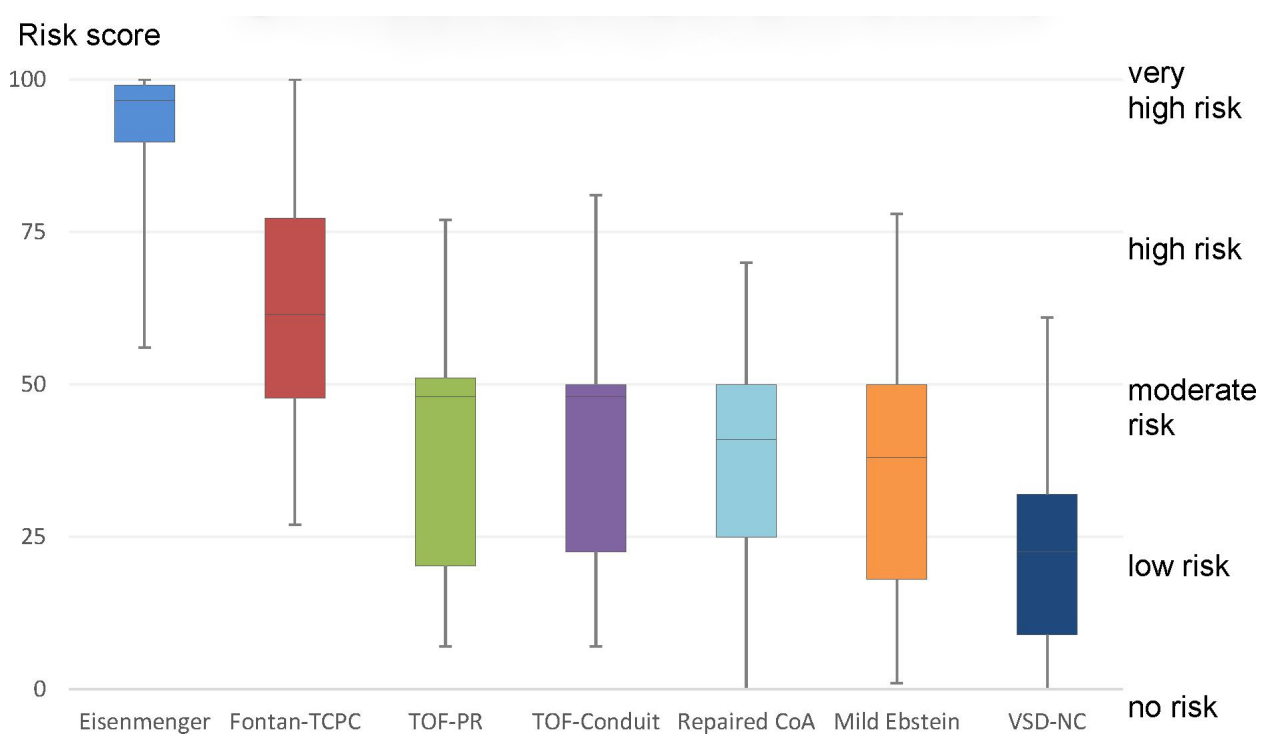

Figure 3 Risk stratification (poor COVID-19 outcome) among seven possible ACHD scenarios. ACHD, adult with congenital heart disease; Fontan-TCPC, univentricular physiology with total cavopulmonary connection and good haemodynamics; repaired CoA, repaired aortic coarctation with mild residual hypertension; TOF-conduit, repaired tetralogy of Fallot with conduit implantation; TOF-PR, repaired tetralogy of Fallot with residual severe pulmonary regurgitation; VSD-NC, repaired ventricular septal defect and mild non-compaction cardiomyopathy. 
cyanotic heart disease were considered at highest risk. There was however a marked disparity among ACHD specialists on risk estimation for the most common clinical scenarios.

ACHD patients currently compose a rather young population. ${ }^{23}$ Due to their age, a milder disease course in case of COVID-19 might be expected when compared with older general population. However, ACHD-specific disorders (eg, restrictive lung disease due to repeated thoracotomies, residual shunts and so on) or associated genetic disorders (ie, 22q11 microdeletion or trisomy of chromosome 21) have the potential to increase their risk for an adverse COVID-19 outcome.

In addition, the heterogeneity of cardiac defects and repair strategies performed in the past makes risk stratification among ACHD patients challenging even for ACHD experts, as illustrated by the disparity of estimated risk among the presented clinical scenarios. Reassuringly, most experts consider the majority of ACHD patients to be at low risk for poor outcomes in case of COVID-19. However, ACHD patients at advanced age and with symptomatic heart failure or immunosuppression, as well as those with pulmonary arterial hypertension, cyanosis and a Fontan circulation, are considered vulnerable in case of COVID-19.

The level of evidence of our research is considered to be lowest when using the classification of the Oxford (UK) Centre for Evidence-based Medicine: Levels of Evidence. ${ }^{9}$ However, until the much-needed outcome studies reflecting the disease course in ACHD patients are available, we may rely on the above-mentioned results not only to assess patient risk but also to reassure many of our patients.

\section{Author affiliations}

${ }^{1}$ Center for Congenital Heart Disease, Cardiology, Inselspital University Hospital Bern, University of Bern, Bern, Switzerland

${ }^{2}$ Division of Cardiology, University Hospital Basel, University of Basel, Basel, Switzerland

${ }^{3}$ University Heart Centre Zurich, Department of Cardiology, University of Zurich, Zurich, Switzerland

${ }^{4}$ Cardiovascular Department, Lausanne University Hospital and University of Lausanne, Lausanne, Switzerland

${ }^{5}$ Service de Cardiologie, University Hospital of Geneva, Geneve, Switzerland

${ }^{6}$ Adult Congenital Heart Disease Unit, Centre de Référence des Malformations Cardiaques Congénitales Complexes, M3C, Inserm U970, Paris Centre de Recherche Cardiovasculaire, Hôpital Europeen Georges-Pompidou Pôle Cardiovasculaire Rénal Métabolique, Paris, France

${ }^{7}$ Unitat Integrada de Cardiopaties Congènites de l'Adolescent i I'Adult, Vall d'Hebron-Sant Pau, Vall d'Hebron University Hospital. Vall d'Hebron Barcelona Hospital Campus and CIBERCV, Barcelona, Spain

${ }^{8}$ Adult Congenital Heart Disease Unit, Instituto de BioMedicina de Sevilla (IBIS) and CIBERCV, Virgen del Rocio University Hospital Cardiology and Cardiovascular Surgery Service, Sevilla, Spain

${ }^{9}$ Adult Congenital Heart Disease Centre, Medical University of Vienna, Wien, Austria

${ }^{10}$ Department of Cardiology, Amsterdam UMC Location AMC, Amsterdam, The Netherlands
Collaborators EPOCH-ASO is funded by internal grants without support from the pharmaceutical industry. We thank the expert panel who (in addition to the coauthors of the manuscript) participated in this survey: Werner Budts (Belgium), Massimo Chessa (Italy), Julie De Backer (Belgium), Gerhard Diller (Germany), Rocío García Orta (Spain), María Elvira Garrido-Lestache Rodríguez-Monte (Spain), Elvira Ana González (Spain), Pablo Meras (Spain), Berta Miranda (Spain), Marielle Morissens (Belgium), Agnes Pasquet (Belgium), Joaquin Rueda Soriano (Spain), Annette Schophuus Jensen (Denmark), Lars Sondergaard (Denmark), Oktay Tutarel (Germany), Annemien van den Bosch (The Netherlands), Heleen van der Zwaan (The Netherlands). For more information about EPOCH (European Collaboration for Prospective Outcome Research in Congenital Heart disease), please visit: https:// www.sacher-registry.com/epoch/.

Contributors FJJR-R, DT, MG and MS contributed to drafting of the manuscript, conception of the research, critical revision of the manuscript for important intellectual content and supervision. ML, PG, LD-S, BB, HG and JB contributed to critical revision of the manuscript for important intellectual content and supervision.

Funding EPOCH-ASO is funded by internal grants without support from the pharmaceutical industry.

Competing interests None declared.

Patient consent for publication Not required.

Ethics approval Because no data on human subjects were reported, ethical/IRB approval for our survey was waived.

Provenance and peer review Not commissioned; externally peer reviewed.

Data availability statement Data are available upon reasonable request.

Open access This is an open access article distributed in accordance with the Creative Commons Attribution Non Commercial (CC BY-NC 4.0) license, which permits others to distribute, remix, adapt, build upon this work non-commercially, and license their derivative works on different terms, provided the original work is properly cited, appropriate credit is given, any changes made indicated, and the use is non-commercial. See: http://creativecommons.org/licenses/by-nc/4.0/.

\section{ORCID iDs}

Francisco Javier Ruperti-Repilado http://orcid.org/0000-0002-9904-0402

Laura Dos-Subira http://orcid.org/0000-0001-5821-4501

Pastora Gallego http://orcid.org/0000-0003-2115-5047

Markus Schwerzmann http://orcid.org/0000-0002-4006-8929

\section{REFERENCES}

1 Control ECfDPa. COVID-19 situation update for the EU/EEA and the UK, as of 20, 2020. Available: https://www.ecdc.europa.eu/en/cases2019-ncov-eueea [Accessed 20 Jun 2020].

2 Oliver Ruiz JM, Dos Subirá L, González García A, et al. Adult congenital heart disease in Spain: health care structure and activity, and clinical characteristics. Rev Esp Cardiol 2020;73:804-11.

3 Tobler D, Schwerzmann M, Bouchardy J, et al. Swiss Adult Congenital HEart disease Registry (SACHER) - rationale, design and first results. Swiss Med Wkly 2017;147:w14519.

4 Greutmann M, Tobler D, Kovacs AH, et al. Increasing mortality burden among adults with complex congenital heart disease. Congenit Heart Dis 2015;10:117-27.

5 Diller G-P, Kempny A, Alonso-Gonzalez R, et al. Survival prospects and circumstances of death in contemporary adult congenital heart disease patients under follow-up at a large tertiary centre. Circulation 2015;132:2118-25.

6 Radke RM, Frenzel T, Baumgartner $\mathrm{H}$, et al. Adult congenital heart disease and the COVID-19 pandemic. Heart 2020;106:1302-9.

7 Alsaied T, Aboulhosn JA, Cotts TB, et al. Coronavirus disease 2019 (COVID-19) pandemic implications in pediatric and adult congenital heart disease. J Am Heart Assoc 2020;9:e017224.

8 Diller G-P, Gatzoulis MA, Broberg CS, et al. Coronavirus disease 2019 in adults with congenital heart disease: a position paper from the ESC Working group of adult congenital heart disease, and the International Society for adult congenital heart disease. Eur Heart J 2020;323.

9 Oxford Centre for Evidence-Based Medicine: Levels of Evidence, March 2009. Available: https://www.cebm.ox.ac.uk/resources/levelsof-evidence/oxford-centre-for-evidence-based-medicine-levels-ofevidence-march-2009 